\author{
Revista Facultad 2021 \\ de Ciencias Básicas Vol. 17(1) \\ enero-junio - ISSN: 1900-4699 - e-ISSN: 2500-5316 - pp.91-100
}

DOI: https://doi.org/10.18359/rfcb.5742

(1) (1) $\Theta(\Theta$

\title{
Caracterización de semillas de tres especies de palmas y su relación con la depredación posdispersiva*
}

\author{
Elsa Helena Manjarres Hernándeza
}

Resumen: el éxito reproductivo de las plantas depende de la dispersión de sus semillas. Uno de los procesos que influye sobre esta dispersión es la depredación. En particular, la depredación posdispersión influye en la supervivencia y la distribución espacial de las plantas. En este trabajo se evaluó la relación de la depredación posdispersión de las semillas de tres palmas, Oenocarpus bataua, Euterpe precatoria y Socratea exorrhiza, con los patrones de denso-dependencia, el tamaño de las semillas y la germinación en un bosque del oriente colombiano. De cada especie de palma se seleccionaron tres individuos, en los que se recolectaron semillas de acuerdo con su patrón de agregación, cuarenta semillas agregadas y cuarenta semillas no agregadas. A las semillas se le determinó el peso, el diámetro, la germinación y la depredación. Se encontró que el tamaño de las semillas fue diferente en las tres especies de palmas, existe una correlación positiva entre el peso y el diámetro de las semillas, los porcentajes de depredación son de aproximadamente el 50 \% y yna relación entre la germinación de las semillas y su depredación. Así, las dinámicas poblacionales de las palmas estudiadas se pueden asociar a características de sus semillas tales como tamaño, germinación, denso-dependencia y su capacidad de resistir a los depredadores.

Palabras clave: depredación posdispersión; Euterpe precatoria; Oenocarpus bataua; Socratea exorrhiza; patrón de agregación

Recibido: 24 de abril de 2021

Aceptado: 2 de septiembre de 2021

Disponible en línea: 19 de noviembre de 2021

Cómo citar: E. H. Manjarres Hernández, «Caracterización de semillas de tres especies de palmas y su relación con la depredación posdispersiva», Rev. Fac. Cienc. Básicas, vol. 17, n. 1, pp. 91-100, nov. 20

* Artículo de investigación.

a Ph. D. (c) en Ciencias Biológicas, M. Sc. en Ciencias Biológicas, bióloga. Universidad Pedagógica y Tecnológica de Colombia, Facultad de Ciencias, Escuela de Ciencias Biológicas, Tunja, Colombia. Correo electrónico: elsa.manjarres@uptc.edu.co oRCID: https://orcid.org/0000-0001-6221-8636 


\title{
Characterization of Seeds of Three Palm Species and their Relationship to Post-Dispersal Predation
}

\begin{abstract}
One of the processes influencing this dispersal is predation. In particular, post-dispersal predation influences the survival and spatial distribution of plants. In this work we evaluated the relationship of post-dispersal predation of seeds of three palms, Oenocarpus bataua, Euterpe precatoria and Socratea exorrhiza, with the patterns of dense-dependence, seed size and germination, in a forest of eastern Colombia. Three individuals were selected from each palm species, from which seeds were collected according to their aggregation pattern, forty aggregated seeds and forty non-aggregated seeds. Seed weight, diameter, germination, and predation were determined. It was found that seed size was different in the three palm species, there was a positive correlation between seed weight and diameter, predation percentages were approximately $50 \%$, and there was a relationship between seed germination and predation. Thus, the population dynamics of the palms under study can be associated with seed characteristics such as size, germination, density-dependence and their ability to resist predators.
\end{abstract}

Keywords: post-dispersal predation; Euterpe precatoria; Oenocarpus bataua; Socratea exorrhiza; aggregation pattern

\section{Caracterização de sementes de três espécies de palmeiras e sua relação com a predação pós-dispersiva}

Resumo: o sucesso reprodutivo das plantas depende da dispersão de suas sementes. Um dos processos que influencia essa dispersão é a predação. Em particular, a predação pós-dispersão influencia a sobrevivência e a distribuição espacial das plantas. Neste trabalho, a relação da predação pós-dispersão de sementes de três palmeiras, Oenocarpus bataua, Euterpe precatoria e Socratea exorrhiza, foi avaliada com padrões de dependência densa, tamanho de semente e germinação em uma floresta no leste da Colômbia. Foram selecionados três indivíduos de cada espécie de palmeira, em que as sementes foram coletadas de acordo com seu padrão de agregação, quarenta sementes agregadas e quarenta sementes não agregadas. As sementes foram determinadas pelo peso, pelo diâmetro, pela germinação e pela predação. Verificou-se que os tamanhos das sementes foram diferentes nas três espécies de palmeiras, há uma correlação positiva entre o peso e o diâmetro das sementes, as porcentagens de predação são de aproximadamente 50 \% e uma relação entre germinação de sementes e predação de sementes. Assim, a dinâmica populacional das palmeiras estudadas pode estar associada às características de suas sementes, como tamanho, germinação, dependência densa e sua capacidade de resistir a predadores.

Palavras-chave: predação pós-dispersão; Euterpe precatoria; Oenocarpus bataua; Socratea exorrhiza; padrão de agregação. 


\section{Introducción}

En los árboles de bosques tropicales, cerca de la mitad de las semillas que estos producen mueren antes de germinar producto de la depredación de animales y el ataque de hongos [1]. La intensa mortalidad de semillas en varios ecosistemas naturales o transformados se evidencia en la escasez de plántulas y árboles jóvenes. Es, entonces, la depredación una de las causas más estudiadas para dicha mortalidad [2]. La depredación, junto con procesos como, por ejemplo, la germinación, la competencia, la colonización y el flujo genético, ejercen una fuerza selectiva en la demografía de las plantas [3], [4].

Algunos estudios que involucran plantas de bosques tropicales comparan la tasa de depredación en diferentes hábitats y estaciones climáticas, de modo que concluyen que los patrones de depredación varían ampliamente entre especies, según el hábitat y las condiciones medioambientales [5]. La depredación de semillas se aborda considerando la depredación predispersión y posdispersión de manera separada, debido a las marcadas diferencias entre los organismos vinculados a cada tipo de depredación [6], [7].

La depredación posdispersión la realizan, principalmente, coleópteros, dípteros, lepidópteros e himenópteros [2]; esta es determinante en la viabilidad y germinación de las semillas en bosques tropicales. Este tipo de depredación influye en la supervivencia y la distribución espacial de las plantas [8], debido a que reduce la distancia a la que las semillas pueden dispersarse para establecerse en un sitio apropiado [1].

Sin embargo, las plantas han desarrollado estrategias dirigidas a evadir el ataque de los depredadores. Una de las estrategias es la cantidad de semillas producidas. Estudios en árboles tropicales muestran que la cercanía de las semillas al árbol parental, generalmente, delimita las pautas de depredación [9]. Por tanto, Janzen señala dos pautas que deben esperarse en la depredación posdispersión: "depredación dependiente de la densidad y depredación dependiente de la distancia” [10]. Por otra parte, investigaciones han mostrado que en los frugívoros, al alejar o remover los frutos del parental, disminuye la mortalidad por factores como la denso-dependencia y la depredación, debido a que se estimula la germinación de las semillas y así logran escapar de los depredadores [11].

En los bosques tropicales, las palmas juegan un papel fundamental, ya que sus flores y frutos son una fuente de alimento para muchos animales, en especial los frutos y las semillas se caracterizan por la composición lipídica y proteica de alto valor nutritivo [12], [11]. Estas características hacen que estas semillas tengan mayor riesgo de depredación [13]. Por tanto, es necesario caracterizar las semillas de las palmas, debido a que estas influyen sobre su depredación posdispersión, su denso-dependencia y la germinación, además de afectar la dinámica de las poblaciones en los bosques tropicales [13], [14].

En este contexto, el presente estudio tuvo como objetivo evaluar la depredación posdispersión de semillas de tres especies de palmas (Oenocarpus bataua, Euterpe precatoria y Socratea exorrhiza) y su relación con los patrones de denso-dependencia, el tamaño de las semillas y la germinación.

\section{Materiales y métodos}

\section{Área de estudio}

El trabajo se llevó a cabo en la reserva biológica El Caduceo, ubicada en el municipio de San Martín (034' $17^{\prime \prime}$ N-7339'34” o), departamento del Meta, Colombia. La reserva presenta una extensión de 142 hectáreas, cuenta con un fragmento de bosque de galería que corresponde a bosque húmedo tropical de sabana ondulada de los Llanos Orientales colombianos, y un área que corresponde a potreros. La zona tiene niveles de precipitación anual entre los 2000-3000 mm, el número de días de lluvia al año varía de 100 a 200 , y la temperatura media anual es de $24{ }^{\circ} \mathrm{C}$ [15]. El estrato arbóreo está compuesto por especies perennifolias y unas pocas caducifolias, alcanzando alturas de $20-30 \mathrm{~m}$ con algunas especies de palmas que en ocasiones sobresalen del dosel. 


\section{Especies de estudio}

Las tres especies de palmas estudiadas fueron $\mathrm{Oe}$ nocarpus bataua, Euterpe precatoria y Socratea exorrhiza. Los sitios donde se encontraron los organismos de estudio difieren entre sí con respecto a la vegetación circundante. Las zonas donde se encontraban $E$. precatoria presentaban una vegetación arbórea abundante y de estratos bajos, representados, principalmente, por hierbas y arbustos; el promedio de altura de estas palmas fue de $17 \mathrm{~m}$ y cada individuo presentó dos inflorescencias en la planta. Los sitios de los individuos de O. bataua exhibían vegetación circundante con dominancia del estrato herbáceo, la altura promedio de las palmas fue de $15 \mathrm{~m}$. Las zonas de los individuos de $S$. exorrhiza son más húmedos con respectos a las de las otras especies, y la altura promedio de las palmas fue de $14 \mathrm{~m}$.

Oenocarpus bataua es una palma ampliamente distribuida y abundante en los bosques tropicales de América del Sur [16]. En Colombia se distribuye en zonas de bosque húmedo de tierras bajas, incluyendo el Piedemonte llanero según estudios de [17]. Es una palma arborescente, monoica y alógama. Los frutos se caracterizan por la composición lipídica y proteica de alto valor nutritivo; cuando maduran son negro-violáceos, cada palmera produce entre tres y cuatro racimos y cada racimo tiene más de mil frutos. Se han reportado cinco especies de mamíferos que dispersan los frutos sin dañar la semilla (Sciurus granatensis, Microsciurus mimulus, Dasyprocta punctata, Proechimys sp. y Marmosa robinsoni) [11].

Socratea exorrhiza es una palma solitaria, alcanza hasta $20 \mathrm{~m}$ de altura. Sus raíces sobresalen del piso formando un cono hasta de $3 \mathrm{~m}$ de alto y está cubierta de espinas. Presenta inflorescencia emergiendo por debajo de las hojas, pedúnculo hasta $46 \mathrm{~cm}$ de largo y raquis hasta de $20 \mathrm{~cm}$ de largo. Fruto elipsoide a ovoide, color café-amarillento, semilla obovada a oblonga, de superficie irregular [17], [18]. Los dispersores que se han reportado para la especie son mamíferos terrestres como los pecaríes de collar (Pecari tajacu); en Centroamérica se han reportado especies como Dasyprocta punctata [19].
Euterpe precatoria es una palma solitaria, se encuentra en lugares con periodos cortos anuales de inundación y llega a alcanzar alturas de entre 20-30 m, así como diámetros de $15-20 \mathrm{~cm}$. Presenta raíces de color rojizo, un estípite liso, los frutos presentan forma globosa y alcanzan diámetros de hasta $1,8 \mathrm{~cm}$ de color púrpura que, al madurar, contienen una semilla de color negro [20]. Estudios han reportado pequeños roedores, así como aves del género Tardus, como dispersores de semillas de esta especie de palma [21].

\section{Caracterización y depredación de semillas}

Se definieron dos estados de densidad o patrones de agrupación de las semillas: semillas agregadas y semillas no agregadas. Se consideraron semillas agregadas a las que se encontraban en grupos de más de cuarenta, alejadas entre sí un máximo de $5 \mathrm{~cm} \mathrm{y}$, por lo general, cercanas al eje parental. Las semillas no agregadas fueron aquellas que se hallaban alejadas entre sí una distancia mayor a $10 \mathrm{~cm}$, además de que se encontraban lejos del parental en un área circular con radio no mayor a $3 \mathrm{~m}$.

De cada especie de palma se seleccionaron aleatoriamente tres individuos, de modo que se realizó la colecta de las semillas en el suelo, de acuerdo con su patrón de agrupación; aproximadamente, cuarenta semillas agregadas y cuarenta semillas no agregadas. Al final se obtuvieron 249 semillas de S. exorrhiza, 237 de O. bataua y 240 de E. precatoria.

A cada semilla recolectada se le realizaron mediciones de diámetro, peso, depredación y estado de germinación. El estado de germinación se determinó observando la presencia de radícula en la semilla. A fin de establecer la depredación, a las semillas se les retiró cualquier resto de cáscara y/o pulpa y se realizó una inspección externa en busca de agujeros o fisuras en la testa, así como de cualquier otra condición que revelara depredación. Luego se procedió a abrir las semillas para verificar el estado interno y el depredador. Los depredadores encontrados fueron conservados en alcohol para su determinación. 


\section{Análisis de datos}

Con los datos obtenidos de peso y diámetro de las semillas se realizó un análisis descriptivo, prueba de homogeneidad de varianzas de Levene y prueba de normalidad de Shapiro-Wilk. Adicionalmente, se realizaron análisis de correlación de Pearson y análisis de regresión simple entre el peso y el diámetro de las semillas usando el programa R y las librerías psych, Hmisc y corrplot [22]. En cuanto a las variables depredación, estados de densidad y germinación de semillas, se desarrollaron tablas de frecuencias y contingencia, se generaron los coeficientes v de Cramer y el coeficiente de contingencia usando el programa estadístico Infostat [23]. Se realizó la prueba Chi cuadrado de bondad de ajuste con un nivel de confianza del $95 \%$ para los patrones de densidad de las semillas, de germinación y de depredación. Con respecto a la relación entre el tamaño de las semillas y la depredación se realizó la prueba $\mathrm{T}$ con un nivel de confianza del $95 \%$, usando el programa estadístico Infostat [23]. Por último, se generó una gráfica de barras de error para el peso y la depredación mediante el programa $\mathrm{R}$ utilizando el paquete ggplot2 [24].

\section{Resultados y discusión}

En las tres especies de palmas estudiadas, cada fruto produce una sola semilla, por tanto, las variaciones en el tamaño van a depender directamente de las condiciones ambientales. Minor y Kobe plantean que las variaciones en el tamaño de las semillas pueden resultar de las diferencias entre las plantas adultas tales como la disponibilidad de nutrientes y agua, o de la cantidad de tejido fotosintético [25]. El peso y el diámetro de las semillas son variables usadas para la estimación del tamaño de las semillas [13]. En este caso las semillas de S. exorrhiza presentaron pesos entre $4 \mathrm{a} 14 \mathrm{~g}$ y un diámetro promedio de $20,63 \mathrm{~mm}, O$. bataua pesos entre 2 y $10 \mathrm{~g}$ y diámetro promedio de $19,16 \mathrm{~mm}, \mathrm{y}$ E. precatoria pesos cercanos a $1 \mathrm{~g}$ y diámetro promedio de 10,89 mm (véase la Tabla 1). La característica más variable fue el peso en las tres especies de palmas con coeficientes de variación mayores al $20 \%$.

Al establecer la correlación entre el diámetro y el peso de las semillas de las tres especies de palmas se observó una relación positiva y significativa entre estas dos variables (correlación de Pearson $=0,89$ ). Adicionalmente, los análisis de regresión lineal mostraron que el tipo de relación entre el peso y el diámetro de semillas se ajustó de manera significativa $(p<0,05)$ a un modelo lineal simple y un comportamiento normal de los datos (véase la Figura 1).

Esta correlación positiva observada, posiblemente, se debe a que todas las semillas presentaron estructuras compactas, por tanto, si hay variación en el diámetro habría un cambio en el peso. Mientras que la depredación puede ocasionar disminución en el peso de las semillas por la pérdida en los tejidos, sin afectar su diámetro.

Si consideramos la reducción en el tamaño de las semillas como una estrategia para cumplir las exigencias regenerativas de las especies [1], se puede reconocer que E. precatoria se comporta como una especie pionera debido a que presenta un

Tabla 1. Estadísticos descriptivos para peso $(\mathrm{g})$ y diámetro $(\mathrm{mm})$ de semillas de las tres especies de palmas

\begin{tabular}{lcccccccc}
\hline Especie & Variable & $\mathbf{n}$ & Media & Mínimo & Máximo & DE & CV (\%) \\
\multirow{2}{*}{ S. exorrhiza } & Peso & 249 & 7,74 & 4,00 & 14,00 & 2,70 & 34,87 \\
& Diámetro & 249 & 20,63 & 10,00 & 29,00 & 3,05 & 14,78 \\
0. bataua & Peso & 237 & 5,86 & 2,00 & 10,00 & 1,72 & 29,35 \\
& Diámetro & 237 & 19,16 & 10,10 & 23,60 & 1,88 & 9,76 \\
E. precatoria & Peso & 240 & 0,82 & 0,50 & 1,10 & 0,17 & 20,73 \\
& Diámetro & 240 & 10,89 & 8,60 & 13,90 & 0,89 & 8,08 \\
\hline
\end{tabular}

n: número de semillas por especie palma, DE: desviación estándar, Cv: coeficiente de variación.

Fuente: elaboración propia. 

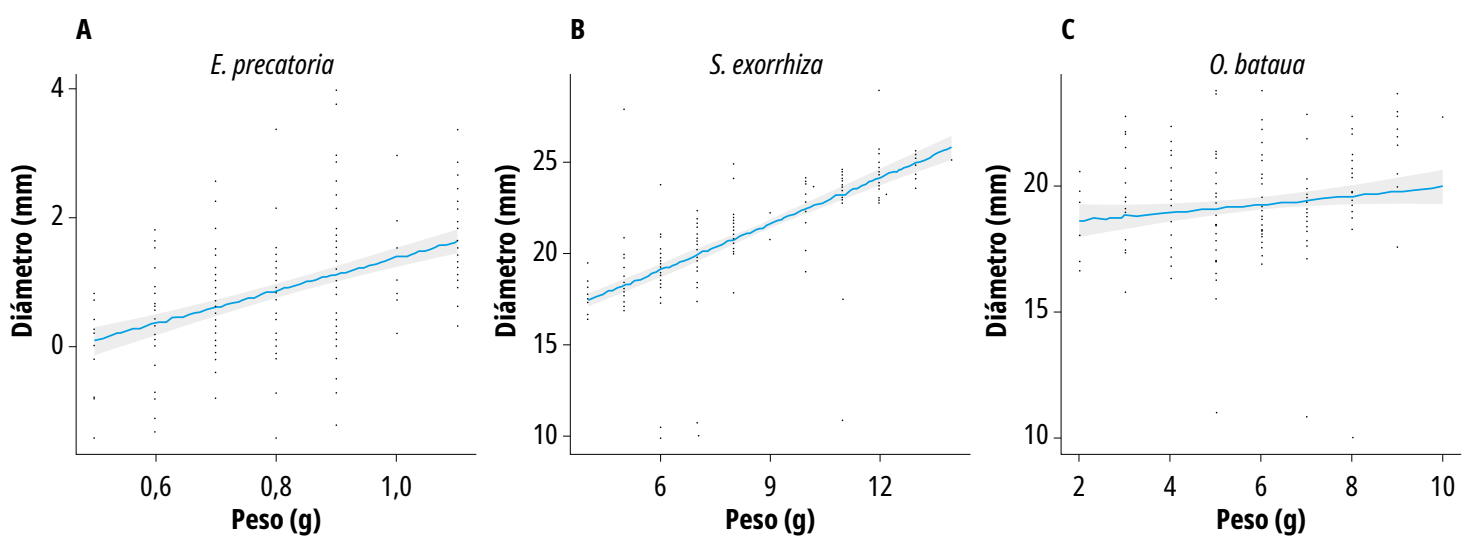

Figura 1. Regresión lineal entre el diámetro $(\mathrm{mm})$ y peso de semillas $(\mathrm{g})$ en las tres especies de palmas. Fuente: elaboración propia.

tamaño reducido de las semillas que le permitiría alcanzar claros de bosque más fácilmente debido a su tamaño. Por otra parte, se ha demostrado que especies con semillas grandes producen plantas vigorosas, mientras las pequeñas se deben adaptar a la colonización de nuevos espacios [26].

Los estados de agregación de las semillas y la depredación no mostraron diferencias significativas $(p=0,75 ; p>0,05 ; \mathrm{X} 2=0,57)$. En la Figura 2a se observa que los porcentajes de depredación son de aproximadamente el $50 \%$, independiente del patrón de agregación. En cuanto a la germinación y los patrones de agregación, se evidencia diferencias significativas $(p=0,0001 ; p<0,05$; X2 $=20,84)$; así, las semillas no agregadas mostraron porcentajes menores de germinación con respecto

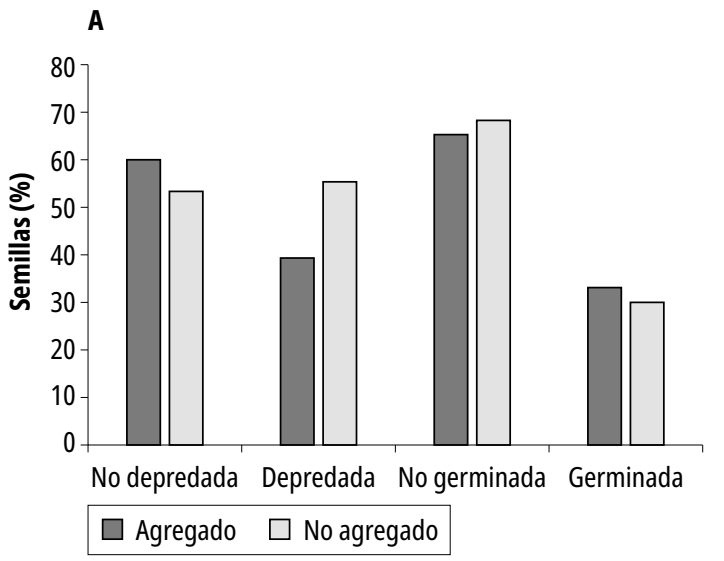

a las semillas agregadas (Figura 2a). Esta relación entre los patrones de agregación y la germinación corrobora que las tres especies de palmas presentan depredación posdispersión dependiente de la densidad de acuerdo con el modelo propuesto por Janzen-Connell [27].

Ahora bien, el $61 \%$ de las semillas de $S$. exorrhiza se encontraron depredadas, le sigue $O . b a$ taua con el $36 \%$ y, por último, E. precatoria con el $31 \%$. Mediante la prueba $\mathrm{T}$ de student se observó diferencias significativas $(p<0,05)$ entre la depredación y el tamaño de semilla, es decir, existe un efecto en el tamaño de semilla y la depredación. Las semillas de S. exorrhiza tienen un mayor tamaño, por tanto, entre más grande sea la semilla el endospermo tiende a ser más depredado (Figura 2b).

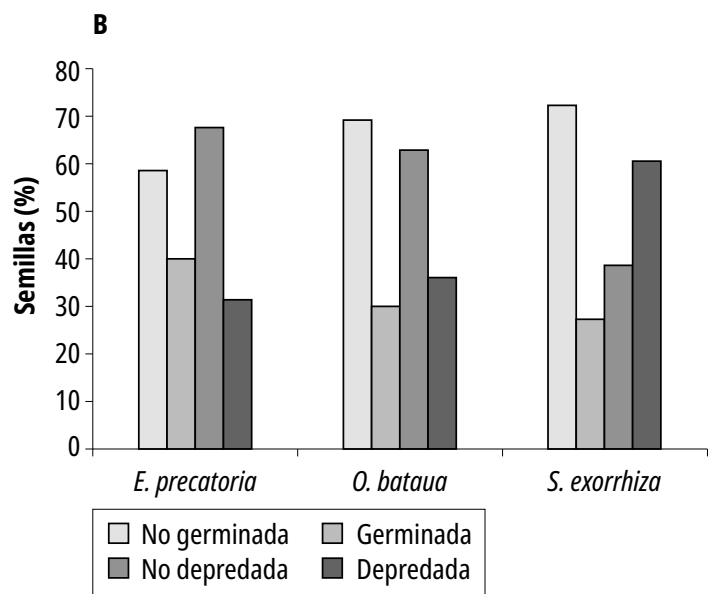

Figura 2. a) relación entre el patrón de agrupación, la depredación y la germinación de las semillas en las tres especies de palmas; b) porcentaje de semillas depredadas y germinadas según la especie de palma. Fuente: elaboración propia. 
Los porcentajes de germinación en las tres especies de palmas fueron bajos; E. precatoria presento un $41 \%$ de semillas germinadas, mientras que en $S$. exorrhiza y $O$. bataua el porcentaje fue del $30 \%$. Además, se observó que existen diferencias entre la germinación y la especie de palma. La baja germinación de las semillas de $S$. exorrhi$z a$ probablemente se debió a que un gran número de semillas estaban depredadas. Según Martínez et al., la germinación depende del estado de las semillas después de estar expuestas a los ataques que sufren de patógenos y depredadores [28]. En algunos casos, fue posible observar germinación de las semillas, pese a haber sido atacadas por depredadores, demostrando una posible adaptación a los ataques producidos, principalmente, por los insectos, tal como lo evidencian DeSoto et al. [29].

Por otra parte, la depredación del endospermo sin afectar el embrión es un factor determinante para la germinación de semillas depredadas. En el caso de las semillas no germinadas y no depredadas, los bajos porcentajes de germinación pueden deberse, entre otras causas, a que estas especies presentan semillas recalcitrantes, por tanto, requieren de las condiciones adecuadas para germinar. Asimismo, las semillas de estas palmas requieren de procesos de escarificación para su pronta germinación [30].

A fin de que la germinación se efectúe y se manifieste en el reclutamiento de individuos en una población, es necesario tener en cuenta factores ambientales y características propias de los micrositios de las semillas [31]. Estudios muestran que en los micrositios con vegetación densa el fracaso en el reclutamiento de las plantas se debe más a la presión de la depredación que a la competencia interespecífica con dicha vegetación [32]. Por otra parte, la calidad de las semillas y las estrategias de las plantas para su dinámica reproductiva influyen también en los niveles de germinación [1]. En el caso de las palmas, además de estas limitaciones, factores ambientales y los micrositios, los eventos históricos y denso-dependientes pueden causar distribución agrupada en los diferentes ambientes, de modo que afectan igualmente el reclutamiento de individuos en una población.
La gran diversidad de insectos depredadores muestra que el tamaño de las semillas no es por sí mismo un método efectivo para evadir la depredación, aunque insectos particulares pueden evitar semillas grandes o pequeñas [33]. La relación de la depredación con el peso de las semillas mostró que cuando aumenta la depredación el peso disminuye (véase la Figura 3). E. precatoria presentó menores porcentajes de depredación, lo cual, posiblemente, se asocia a que sus semillas son pequeñas y poseen menor cantidad de endospermo que pueda ser consumido. Esta relación coincide con el patrón encontrado por [6], en el cual las semillas disminuyen su peso pero aumentan su número buscando saciar al depredador.

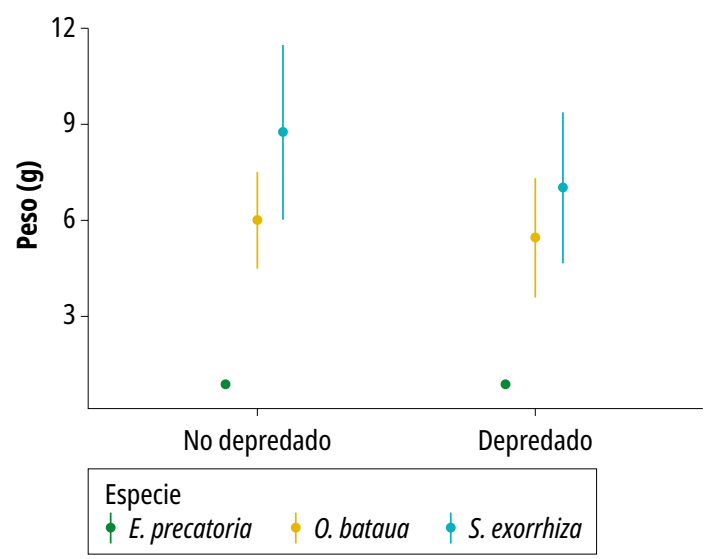

Figura. 3. Promedio del peso de las semillas cuando presentan depredación y sin depredación en las tres especies de palmas. Fuente: elaboración propia.

Las semillas de mayor tamaño, como, por ejemplo, las de S. exorrhiza, presentaron mayor depredación, lo cual indicaría que los depredadores en esta región tienen preferencia por las semillas de gran tamaño, tal como lo señala Janzen [10], dada la cantidad de energía que estas semillas le pueden ofrecer, sobre todo para las hembras, las cuales necesitan mayor cantidad de alimento para garantizar el desarrollo de sus larvas [34].

Las especies S. exorrhiza y O. bataua presentaron características similares en tamaño y consistencia, pero se relacionan de manera diferente con la depredación, lo que evidenciaría dos estrategias distintas frente al ataque de los depredadores. Se 
encontró, además, que esta relación entre la depredación y el peso es más marcada para las semillas no agregadas de S. exorrhiza con respecto a las agregadas. Esto permite deducir que los depredadores podrían estar ejerciendo una selección sobre los tamaños de los frutos, lo cual puede ser el resultado de la preferencia sobre el tipo de nutrientes, como, por ejemplo, los lípidos y las proteínas que ofrece cada semilla durante las diferentes etapas de su desarrollo.

Sin embargo, Brewer reportó que la depredación en la palma Astrocaryum mexicanum no estaba relacionada con el tamaño de la semilla y cómo los insectos que depredan las semillas de esta especie parecen no discriminar por tamaño, pero su acción sí dificulta o detiene el desarrollo de la semilla [35].

Los insectos depredadores encontrados en las tres especies de palmas fueron coleópteros pertenecientes a la familia Scolytidae, la cual comprende trece subfamilias, aproximadamente 180 géneros con unas 6000 especies [36]. Estos insectos de pequeño tamaño, forma cilíndrica o semiesférica, constituyen uno de los grupos de coleópteros xilófagos y espermatófagos de mayor importancia en cuanto plagas secundarias [37]; en este estudio estos coleópteros se encontraron dentro de las semillas de las tres especies de palmas en estados larvales y adultos.

Las especies de brúquidos se caracterizan porque parte de su ciclo de vida ocurre dentro de las semillas [38]. Consumen el endospermo mientras ocurre el desarrollo de sus larvas, hasta su adultez. Generalmente, estos insectos se alimentan durante su ciclo de vida de una sola semilla. El tamaño de los frutos y de las semillas genera un efecto en las tasas de ovoposición y depredación de semillas por parte de estos insectos, pues esto genera mejores ambientes para el desarrollo de las larvas [39].

Finalmente, se observa que características de las semillas tales como tamaño, capacidad de germinación y estado de agregación influyen sobre el éxito reproductivo de las plantas en los bosques tropicales.

\section{Conclusión}

La depredación posdispersión en las tres especies de palmas (Oenocarpus bataua, Euterpe precatoria y Socratea exorrhiza) determina el éxito del establecimiento de las plántulas en los bosques tropicales colombianos, debido a la relación que existe entre la depredación, los patrones de agregación (denso-dependencia) y la germinación de las semillas. En este estudio, el depredador de las semillas es común en las tres especies de palmas. En el caso de las semillas que sufrieron depredación presentaron menores porcentajes de germinación, independiente del patrón de agregación. Sin embargo, semillas en las que la depredación no afectó el embrión lograron germinar. Así, las dinámicas poblacionales de las palmas estudiadas se pueden asociar a las características de sus semillas como, por ejemplo, tamaño, capacidad de germinación y resistencia a los depredadores.

\section{Agradecimientos}

A la Universidad Nacional de Colombia, Facultad de Ciencias, Departamento de Biología, por el préstamo de los laboratorios para la fase experimental. A la Universidad Pedagógica de Colombia, Facultad de Ciencias, Escuela de Ciencias Biológicas y a los revisores por sus valiosos aportes al escrito.

\section{Conflictos de intereses}

El manuscrito fue preparado y revisado con la participación de todos los autores, quienes declaramos que no existe conflicto de intereses que ponga en riesgo la validez de los resultados presentados. En cuanto a la financiación, esta investigación se realizó en la Universidad Nacional de Colombia.

\section{Referencias}

[1] M. Guariguata, G. Kattan, Ecología y conservación de bosques neotropicales. Catargo, Costa Rica: Editora LUR, 2002. 
[2] M. Bogdziewicz, R. Bonal, J. Espelta, M. Kalemba, M. Steele, R. Zwolak, Invasive oaks escape pre-dispersal insect seed predation and trap enemies in their seeds, Inte Zool, vol. 13. n. ${ }^{\circ}$, pp. 228-237, 2018, doi: 10.1111/1749-4877.12285

[3] C. Costa, K. Pivetta, G. De Souza, R. Mazzini, S. Pereira, M. Nogueira, Effects of temperature, light and seed moisture content on germination of Euterpe precatoria palm, Am J Plant Sc, vol. 9. n. ${ }^{\circ}$ 1, pp. 98-106, 2018, doi: 10.1146/annurev.es.25.110194.001403

[4] H. Nonogaki, Seed germination and dormancy: The classic story, new puzzles, and evolution, J Integ Plant Biol, vol. 61. n. ${ }^{\circ}$ 5, pp. 541-563, 2019, doi: 10.1111/ jipb.12762

[5] A. Hargreaves, E. Suárez, K. Mehltreter, I. Myers, S. Vanderplank, H. Slinn, R. Almazán, Seed predation increases from the Arctic to the Equator and from high to low elevations, $S c A d v$, vol. 5. n. 2 , pp. eaau4403, 2019, doi: 10.1126/sciadv.aau4403

[6] F. Worthy, P. Hulme, Scale dependence shapes how plant traits differentially affect levels of pre- and post-dispersal seed predation in Scots pine, Euro $J$ For Res, vol. 138. n. ${ }^{\circ}$, pp. 653-672, 2019, doi: 10.1007/ s10342-019-01198-y

[7] B. Wang, Seed density affects post-dispersal seed predation: evidence from a seed removal experiment of 62 species, Inte Zoo, vol. 15. n. ${ }^{\circ}$, pp. 135-143, 2020, doi: 10.1111/1749-4877.12421

[8] A. Moretti, F. Olguin, M. Pinazo, F. Gortari, J. Vera, C. Graciano, Supervivencia y crecimiento de un árbol nativo maderable bajo diferentes coberturas de dosel en el Bosque Atlántico, Misiones, Argentina, Ec Aus, vol. 29. pp. 099-111, 2019, doi: 10.25260/EA.19.29.1.0.779

[9] J. Rodríguez, C. Aguilar, J. Rodríguez, Patrones espaciales en la dispersión primaria de semillas de Juglans jamaicensis C. DC. subsp. Jamaicensis, Rev Cub Cien For, vol. 6. n. ${ }^{\circ}$ 2, pp. 172-183, 2018.

[10] D. Janzen, Herbivores and the number of tree species in tropical forest, Am Natura, vol. 104, n. ${ }^{\circ} 940$, pp. 501$528,1970$.

[11] J. Franco, R. Rojas, Frugivoría y dispersión de semillas de la palma Oenocarpus bataua en dos regiones con diferentes estados de conservación, Act Biol, vol. 37. pp. 33-45, 2015. [En línea]. Disponible en: https://revistas. udea.edu.co/index.php/actbio/article/view/329005

[12] R. Rojas, S. Gary, Y. Muñoz, Frugivoría y dispersión de semillas de la palma Oenocarpus bataua (Arecaceae) en un bosque de los Andes colombianos, Rev Bio Trop, vol. 60. n. ${ }^{\circ}$ 4, pp. 1445-1461, 2012, doi: 10.15517/RBT. V60I4.2054
[13] I. Hernández, D. Guitián, V. González, Efectos del tamaño de semilla y escarificación del endocarpio sobre la germinación de Mauritia flexuosa (Arecaceae), Act Bot Ven, vol. 40. n. ${ }^{\circ}$, pp. 97-118, 2017.

[14] J. Sánchez, M. Pernús, Y. Torres, D. Barrios, Dormancia y germinación en semillas de árboles y arbustos de Cuba: implicaciones para la restauración ecológica, Ac Bot Cub, vol. 218. n. 2, pp. 77-108, 2019. [Internet]. Disponible en: http://repositorio. geotech.cu/jspui/handle/1234/3953

[15] L. Yañez, N. Vargas, I. Forero, P. Locano, J. Ruiz, Evaluación rápida de las familias y grupos trófico de aves asociadas a sabanas y bosques de galería en la Reserva El Caduceo, La María (San Martín, Meta, Colombia), Mutis, vol. 11. n. ${ }^{\circ}$ 2, 2021, doi:10.21789/22561498.1705

[16] M. Cotos, I. Hameed, S. Escajadillo, E. Llica, M. Figueroa, J. García, Macronutrients, Polyphenols and antioxidant capacity of the peel and pulp of the fruit Oenocarpus bataua Mart."Ungurahui”, Res J Phar Tech, vol. 13. n. ${ }^{\circ}$, pp. 2192-2198, 2020, doi:10.5958/0974360X.2020.00394.7

[17] G. Galeano, R. Bernal, Palmas de Colombia, guía de campo. Bogotá, Colombia: Universidad Nacional de Colombia, Facultad de Ciencias, Instituto de Ciencias Naturales, 2010.

[18] J. A. López, R. Bernal, G. Galeano, Management of Lepidocaryum tenue and Socratea exorrhiza, two Amazonian palms used for thatching, Ethnobot Res App, vol. 19. pp. 1-15, 2020, doi:32859/era.19.49.1-15

[19] L. Cao, Z. Wang, C. Yan, J. Chen, C. Guo, Z. Zhang, Differential foraging preferences on seed size by rodents result in higher dispersal success of medium-sized seeds, Eco, vol. 97. n. ${ }^{\circ}$ 11. pp 3070-3078, 2016, doi: 10.1002/ecy. 1555

[20] M. A. Romayna, W. O. Chávez, A.O. Mallqui, Evaluation of pregerminative treatments on seeds of Euterpe precatoria Mart. (Huasaí) in the city of Pucallpa-Peru, Rev Cub Cien For, vol. 8. n. 1 pp. 88-103, 2020.

[21] A. Da Silva, R. Da Rosa Silveira, A. Aumond, A. Da Silveira, C. Cademartori, Frugivoria e dispersão de sementes de Euterpe edulis mart. (Arecaceae) por mamíferos e aves siilvestres na Mata Atlântica do Sul do Brasil, Rev Bra Zoociên, vol. 18. n. 3 . 2017, doi:10.34019/2596-3325.2017.v18.24681

[22] R. Team, R: A language and environment for statistical computing. Vienna, Austria: R Foundation for Statistical Computing, 2020.

[23] J. A. Di Rienzo, F. Casanoves, M.G. Balzarini, InfoStat versión 2020. Argentina: Centro de Transferencia InfoStat, FCA, Universidad Nacional de Córdoba, 2020. 
[24] H. Wickham, Ggplot2: Gráficos elegantes para el análisis de datos, 2a ed. Nueva York: Springer, 2009, doi:10.1007/978-0-387-98141-3

[25] D. M. Minor, R. K. Kobe, Fruit production is influenced by tree size and size-asymmetric crowding in a wet tropical forest, Eco Evo, vol. 9. n. ${ }^{\circ}$ 3, pp. 1458-1472, 2019, doi:10.1002/ece3.4867

[26] J. Steiner, T. L. Springer, “Seed production”, en Forages: The Science of Grassland Agriculture, pp. 581-592, 2020, doi:10.1002/9781119436669.ch32

[27] P. Marchand, L. Comita, S. Wright, R. Condit, S. Hubbell, N. Beckman, Seed-to-seedling transitions exhibit distance-dependent mortality but no strong spacing effects in a Neotropical forest, Eco, vol. 101, n. ${ }^{\circ}$ 2, e02926, 2020, doi:10.1002/ecy.2926

[28] Y. Martínez, S. Castillo, A. Orozco, J. A. Zavala, L. Bonilla, Micrositios seguros para la germinación de Symphoricarpos microphyllus (Caprifoliaceae), una especie arbustiva con latencia, Act Bot Mex, vol. 126, 2019, doi:10.21829/abm126.2019.1458

[29] L. Desoto, D. Tutor, R. Torices, S. Rodríguez, C. Nabais, Pre-dispersal predation effect on seed packaging strategies and seed viability, Oecol, vol. 180. n. ${ }^{\circ} 1 \mathrm{pp}$. 91-102, 2016, doi:10.1007/s00442-015-3446-8

[30] A. Coronado, A. González, A. Candre, I. Pinto, E. Miraña, A. Bernilla, Estudio de viabilidad y conservación de semillas de cinco especies vegetales amazónicas como base para la creación de un banco de germoplasma en el municipio de Leticia, Amazonas, Colombia, Fol Ama, vol. 27. n. ${ }^{\circ}$ 1. pp 9-23, 2018, doi:10.24841/fa. v27i1.435

[31] V. Iralu, H. S. Barbhuyan, K. Upadhaya, Ecology of seed germination in threatened trees: a review, En Eco Envi, vol. 4. pp. 189-210, 2019, doi: 10.1007/s40974019-00121-w
[32] E. Gasser, M. Schwarz, A. Simon, P. Perona, C. Phillips, J. Hübl, L. Dorren, A review of modeling the effects of vegetation on large wood recruitment processes in mountain catchments, Earth-Sc R, vol. 194. pp. 350-373, 2019, doi: 10.1016/j.earscirev.2019.04.013

[33] P. J. Garrote, G. Calvo, M. Żywiec, M. Delibes, A. Suárez-Esteban, A. Castilla, J. Fedriani, Extrinsic factors rather than seed traits mediate strong spatial variation in seed predation, Pers Plant Eco Ev System, vol. 38. pp. 39-47, 2019, doi: 10.1016/j.ppees.2019.02.005

[34] M. A. Cuny, J. Traine, C. Bustos-Segura, B. Benrey, Host density and parasitoid presence interact and shape the outcome of a tritrophic interaction on seeds of wild lima bean, Scien Rep, vol. 9. n. 1 pp. 1-9, 2019, doi:10.1038/s41598-019-55143-5

[35] S. W. Brewer, Predation and dispersal of large and small seeds of a tropical palm, Oikos, vol. 92 . pp. 245255, 2001, doi: 10.1034/j.1600-0706.2001.920206.x

[36] D. E. Bright, A taxonomic monograph of the bark and ambrosia beetles of the West Indies (Coleoptera: Curculionoidea: Scolytidae). Studies on West Indian Scolytidae (Coleoptera), Insec Mundi, pp. 664, 2018.

[37] M. Arguedas, Pest status and management in the forest plantations of Costa Rica, en Forest pest and disease management in Latin America, pp. 197-210, 2020, doi: 10.1007/978-3-030-35143-4_13

[38] M. Zampetti, L. Toma, Description of seven new species of seed beetles (Coleoptera Bruchidae) from Mediterranean basin and Africa, Bio J, vol. 11 pp 553-564, 2020, doi: 10.31396/Biodiv.Jour.2020.11.2.553.564

[39] I. Martínez, J. Romero, S. Niño, U. Sánchez, F. Sandoval, A. Ventura, Brúquidos (Bruchidae) colectados en el cañón El Novillo y La Libertad en el noreste de México, Ento Mex, vol. 4. pp 513-517, 2017 